\title{
A Fairness Model for Resource Allocation in Wireless Networks
}

\author{
Huaizhou Shi, R. Venkatesha Prasad, Vijay S. Rao, and I.G.M.M. Niemegeers \\ Wireless and Mobile Communications, Faculty of EEMCS, \\ Delft University of Technology, The Netherlands
}

\begin{abstract}
In wireless networks many nodes contend for available resources creating a challenge in resource allocation. With shared resources, fairness in allocation is a serious issue. Fairness metrics have been defined to measure the fairness level of resource decisions in allocations. Therefore, fairness metrics significantly influence network and node performances emphasizing the need for due diligence to fairness metrics. It can be seen that fairness metrics in many of the allocation strategies and algorithms in the literature are not analyzed in depth to show the overall fairness of allocations from different perspectives. Hence, we propose a series of fairness metrics for resource allocation in wireless networks, which evaluate individual, system, short and long term fairness. Our metrics are general enough to be adapted in either multiple or single resource sharing scenarios. Algorithms using these metrics for channel allocation problem in peer-to-peer wireless regional area network is proposed and simulated, and the results show that our metrics in conjunction with smart channel allocation strategies guarantee both the fairness and performances.
\end{abstract}

Keywords: Fairness metrics, Resource allocation, Wireless networks, Jain's index, P2PWRAN.

\section{Introduction}

In wireless networks, many resources have to be shared. While in an individual wireless device or node, its computing resources and memory are shared among different applications, at the network level different layers of the device have to operate in synergy with other nodes in order to achieve successful communication and task completion. In a wireless network, channels, time slots, bandwidth, and other resources need to be allocated to different devices in either centralized or distributed way. Hence, resource allocation plays an important role in wireless networking.

Two optimization goals of resource allocation are utility and fairness [6. Utility reflects the performance of wireless network, and fairness indicates the balance in resource sharing by different wireless devices. Much work can be seen in literature in this regard with emphasis of fairness in resource allocation [7,6]. Though a detailed discussion on fairness issues for resource allocation in wireless networks covering most of the fairness issues is in [2, 7], there is still a lack of general fairness model. Some issues still need more considerations, for example, 
not treating fairness as an independent issue [6], confusing it with resource allocation itself or utility [9], and only considering absolute fairness during allocation in which weights are not adopted or every individual is assigned with the same weight [2, 7, 3].

In this paper, we discuss related work on fairness metrics in wireless networks in Section 2. We propose a new fairness model for resource allocation that contains a series of fairness metrics in Section 3 . These metrics provide an entire view of fairness with multiple fairness metrics. The simulations in Section 4 confirm that our metrics can be employed in resource allocation in wireless networks easily. Conclusions are provided in Section 5 .

\section{Related Work on Fairness Issues}

Some commonly adopted fairness metrics in wireless networks in the literature are Jain's index, max-min and proportional fairness [2,7, 3]. Jain's index does not consider the individual fairness. Another shortcoming is that it cannot be adopted in multiple resource allocation scenarios. Moreover, weighted strategies are not considered in it, and all individuals are treated similarly during allocations, which is hardly true in reality. Max-min indicates a decision 0,1 whether the allocation is fair or not ( 1 implying fair allocation). Thus, max-min fairness cannot measure the level of unfairness. Also, it cannot distinguish between system and individuals. Similar to Jain's index, it is an absolute fairness metric without weighted strategies and individual fairness. Modified max-min fairness are proposed (for example in [1]) to add weights, but they cannot measure the individual fairness. Proportional fairness, however, cannot measure how unfair the system is, and no weighted strategies are considered originally. An advanced proportional fairness metric $(p, \alpha)$-proportional fairness was proposed in $[5]$. Weights of different resources for an individual are added in this model. However, it cannot measure both individual and system fairness. Moreover, assigning the weights to individuals was not described in detail.

Considering the shortcomings in these existing fairness metrics, we try to propose a fairness model which includes a series of fairness metrics for resource allocation that can measure the fairness of a resource allocation decision in the aspects of system, individual, short, and long term fairness.

\section{Fairness Model}

We assume that there are $m(m \geqslant 1)$ different resources, and the capacity of resource $k$ is $C_{k}^{(t)}$. $n$ individuals are in the system. The allocation decision at time $t$ is $\mathbb{X}^{(t)}=\left(X_{1}^{(t)}, \ldots, X_{n}^{(t)}\right)$, in which $X_{k}^{(t)}$ is the allocation of resource $k$ and $X_{k}^{(t)}=$ $\left(x_{k 1}^{(t)}, \ldots, x_{k n}^{(t)}\right) . x_{k i}^{(t)}$ is the amount of resource $k$ that is allocated to individual $i$. Furthermore, $F_{k}^{(t)}\left(\mathbb{X}^{(t)}\right)$ is the system fairness metric on resource $k$ and $f_{k i}^{(t)}\left(\mathbb{X}^{(t)}\right)$ is the fairness metric for an individual $i$ for resource $k . f_{i}^{(t)}$ is the fairness of individual $i$ on all resources. When $m=1$, it is single resource allocation and 
we just drop the resource subscript, for example, $C^{(t)}$ is the resource capacity, $\mathbb{X}^{(t)}=\left(x_{1}^{(t)}, \ldots, x_{n}^{(t)}\right)$ is the allocation decision for an individual $i$. We consider two elements during the weight assignment, which are request ratios and historical allocation information. For individuals with more requests and less historical allocation than others, heavier weights should be assigned to them, giving them higher chance to obtain more resources. Let $\mathbb{R}^{(t)}=\left\{R_{1}^{(t)}, \ldots, R_{m}^{(t)}\right\}$ stand for request at time $t$, in which $R_{k}^{(t)}$ is the allocation request for resource $k . R_{k}^{(t)}=$ $\left(r_{k 1}^{(t)}, \ldots, r_{k n}^{(t)}\right)$ and $r_{k i}^{(t)}$ is the request of resource $k$ from individual $i$ at time $t$. We assume that $x_{k i}^{(t)} \leqslant r_{k i}^{(t)} \leqslant C_{k}$. We also assume the weights are $\mathbb{W}^{(t)}=$ $\left\{W_{1}^{(t)}, \ldots, W_{m}^{(t)}\right\}$ and $W_{k}^{(t)}=\left(w_{k 1}^{(t)}, \ldots, w_{k m}^{(t)}\right)$, in which $W_{k}^{(t)}$ is the weights of resource $k$, and $w_{k i}^{(t)}$ is the weight of individual $i$ for resource $k$ at time $t$. We assign the weight $w_{k i}^{(t)}$ as,

$$
w_{k i}^{(t)}= \begin{cases}e^{\left(1-\frac{\sum_{i} r_{k i}^{(t)}}{n r_{k i}^{(t)}}\right)} & \text { If } r_{k i}^{(t)}=0 ; \\ e^{\left(1-K_{r} \frac{\sum_{i} r_{k i}^{(t)}}{n r_{k i}^{(t)}}-K_{h} \frac{n \sum_{t, r_{k i}^{(t)} \neq 0} \frac{x_{k i}^{(t)}}{r_{k i}^{(t)}}}{\sum_{t, i, r_{k i}^{(t)} \neq 0} \frac{x_{k i}^{(t)}}{r_{k i}^{(t)}}}\right)} & \text { Else if } \sum_{t, i} x_{k i}(t)=0 ; \\ \text { Otherwise. }\end{cases}
$$

Where $w_{k i}^{(t)}$ indicates the importance and priority for a resource $k$ set by an individual $i$ at time $t$ by considering the request and historical allocation. $K_{r}$ and $K_{h}\left(K_{r} \leq 0\right.$ and $\left.K_{h} \leq 0\right)$ are the factors for the current request and historical allocation, and $K r+k_{h}=1$. Therefore, $0 \leqslant w_{k i}^{(t)} \leqslant e$. Based on the weights and request, we define "fair ratio" $e_{k i}^{(t)}$ in Eq. (2), which indicates the satisfaction level of an individual to the current allocation.

$$
e_{k i}^{(t)}=\left\{\begin{array}{l}
e^{2} \quad \text { If } r_{k i}^{(t)} w_{k i}^{(t)}=0 ; \\
\frac{\left.e^{\frac{x_{k i}^{(t)}}{r_{k i}^{(t)}}}\right)}{w_{k i}^{(t)}} \text { Otherwise. }
\end{array}\right.
$$

All our following short and long term and individual and system fairness metrics in this paper are based on fair ratios $\left(e_{k i}^{(t)}\right)$. Incase where there is no request or weight is zero, an individual is satisfied with $\left(e_{k i}^{(t)}=e^{2}\right)$, otherwise, the satisfaction depends on the ratio of its allocation and weight.

The short term system fairness metric for resource $k$ at allocation $t$ (can also be a discrete allocation number) is given by Eq. (3) where $0 \leqslant F_{k}\left(X_{k}^{(t)}\right) \leqslant 1$. It is a variant of Jain's index with the weights and can measure fairness even in multiple resources scenarios.

$$
F_{k}^{(t)}\left(X_{k}^{(t)}\right)= \begin{cases}0 & \text { If } \sum_{i} e_{k i}^{(t)}=0 \\ 1 & \text { Else if } \sum_{i} w_{k i}^{(t)} r_{k i}^{(t)}=0 \\ \frac{\left(\sum_{i} e_{k i}^{(t)}\right)^{2}}{n \sum_{i}\left(e_{k i}^{(t)}\right)^{2}} & \text { Otherwise }\end{cases}
$$


The short term individual fairness of individual $i$ on resource $k$ is shown in Eq. (4), which measures the current fairness of one individual over a certain resource, and $0 \leqslant f_{k i}^{(t)} \leqslant 1$, but with the historical allocation information.

$$
f_{k i}^{(t)}= \begin{cases}0 & \text { If } e_{k i}^{(t)}=0 \\ e^{-\frac{\sum_{i} e_{k i}^{(t)}}{n e_{k i}^{(t)}}} & \text { Otherwise. }\end{cases}
$$

The individual fairness of $i$ for all resources is described in Eq. (5), which is the fairness metric for an individual in the whole resource allocation with multiple resources.

$$
f_{i}^{(t)}= \begin{cases}0 & \text { If } \sum_{k} e_{k i}^{(t)}=0 \\ e^{-\frac{\sum_{k, i} e_{k i}^{(t)}}{n \sum_{k} e_{k i}^{(t)}}} \text { Otherwise. }\end{cases}
$$

Similar to short term fairness metrics, system and individual long term metrics are proposed in Eq. (6), (7), and (8), in which the fairness ratios $e_{k i}^{(t)}$ are considered in a long time period instead of a single time slot.

$$
\begin{gathered}
F_{k}\left(X_{k}\right)= \begin{cases}0 & \text { If } \sum_{t, i} e_{k i}^{(t)}=0 \\
1 & \text { Else if } \sum_{t, i} r_{k i}^{(t)}=0 \\
\frac{\left(\sum_{t, i} e_{k i}^{(t)}\right)^{2}}{n \sum_{i}\left(\sum_{t} e_{k i}^{(t)}\right)^{2}} & \text { Otherwise. }\end{cases} \\
f_{k i}= \begin{cases}0 & \text { If } \sum_{t} e_{k i}^{(t)}=0 \\
e^{-\frac{\sum_{t, i} e_{k i}^{(t)}}{n \sum_{t} e_{k i}^{(t)}}} & \text { Otherwise. }\end{cases} \\
f_{i}= \begin{cases}0 & \text { If } \sum_{t, k} e_{k i}^{(t)}=0 \\
e^{-\frac{\sum_{t, k, i} e_{k i}^{(t)}}{n \sum_{t, k} e_{k i}^{(t)}}} & \text { Otherwise. }\end{cases}
\end{gathered}
$$

Our model is proposed keeping in mind multiple resource allocation scenarios. As a special case for single resource allocation, we just need to set $m=1$, and all metrics and the mechanisms stay the same. For distributed scenarios, allocation decisions are made by individuals instead of a centralized allocation manager. Therefore, the metrics only use the local information they collect, and localized fairness and utility can be achieved. The following advantages can be seen in our fairness metric for resource allocation:

- We adopt a weighted strategy and define it by considering the request and historical allocations, which assign priority to individuals due to their requests and historical allocation information.

- Both system and individual fairness can be measured by our metrics, which describe fairness on different aspects. 
- The fair ratio $e_{k i}^{(t)}$ considers the satisfaction of individuals in an allocation, which gives a more precise description of fairness feature than absolute fairness [2, 7, 3].

- Short term and long term fairness can be measured by our metrics, which may be used in different applications.

- Our fairness model is proposed for multiple resource fair allocation in wireless networks; however, it can also be adapted to other diverse scenarios with slight modification.

\section{Simulations and Results}

\subsection{An Example Scenario}

Peer to peer WRAN (P2PWRAN) was proposed in [8]. P2PWRANs support peer to peer communication in a cell thereby increasing the network capacity by many folds. At the same time, a problem in P2PWRANs is how to allocate the channels efficiently and fairly. Channel allocation has two parameters - (i) total number of available channels, and (ii) interference amongst inter-CPE communication 1 . It is not easy to achieve a fair resource allocation at all times. Therefore, we test our definition of fairness metric model in the channel allocation scenario in a P2PWRAN cell.

Two types of communication can be seen in P2PWRAN communication in a cell, which are communication between CPEs and between CPE to BS. The allocation of channels for CPEs can be treated as a multiple resource allocation case. The first type is the channel allocation for CPE to CPE communication and the other type is the channel allocation for CPE to BS (and BS to CPE) communication. Channel allocation in standard WRAN is queue based [4. Hence, we first queue the channel requests from CPEs, then allocate channel sequentially to the request if no interference is expected to occur. Three different mechanisms for queuing channel requests were adopted in the channel allocation for P2PWRAN in our simulations as follows.

- First one (M1) is the absolute fair queuing mechanism, in which the requests are queued considering the channel allocation history of the nodes. CPEs which have received lesser channels have more chance of getting a channel in the current time slot. $M 1$ treats every individual identically, and tries to reach absolute fairness amongst all individuals during channel allocation.

- The second mechanism (M2) is based on our long term individual fairness metric. The long term individual fairness is employed as the basis for queuing. With (M2), CPEs which are not treated fairly in the past would have more opportunities to obtain channels.

- The third mechanism (M3) queues the requests due to the possibilities of the interference that the requests may cause. Since, if the same channel is allocated to two request $\left(r_{i}, r_{j}\right)$ both of which cause less interference than

${ }^{1}$ For a more detailed discussion of channel allocation in P2PWRAN we refer to 8 . 
two other requests $\left(r_{i}^{\prime}, r_{j}^{\prime}\right)$, then it is better to allocate the channel to $r_{i}$ and $r_{j}$. Generally, requests with shorter transmission distances cause lesser interference than requests with longer distances. Therefore, the transmission distances of the requests are used to queue channel requests, and requests with short transmission distances are allocated first. The purpose of M3 is to reuse channels efficiently.

\subsection{Results}

The network performance on channel reuse and node throughput can be seen in Fig. 1. In Fig. 1(a) and 1(b) the queue mechanism based on our fairness metrics lead to the higher channel reuse times for every channel and most of the nodes obtain higher throughput than the absolute fair mechanism and distance based mechanisms. With our metric in $M 2$, node requests are queued considering individual long term fairness and the ratios of node requests and historical allocation decisions, and it leads to the best performance amongst the three mechanisms. We can also see in the figures that fair mechanisms performs better than the distance based mechanisms (M3).

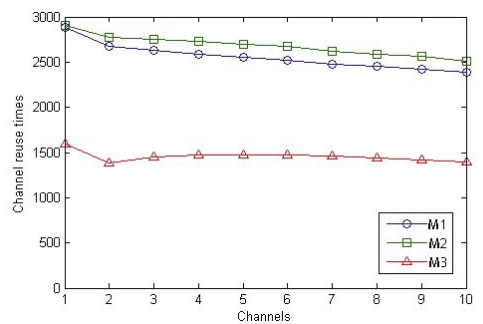

(a) Channel reusing.

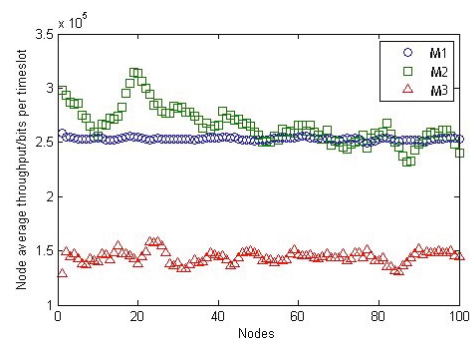

(b) Throughput.

Fig. 1. Performances

The individual fairness of channel allocation is shown in Fig. 2 with both short term (Fig. 2(b) and Fig. 2(d) and long term fairness (Fig. 2(a) and Fig. 2(c) . For CPE to CPE communication, the mechanism based on our metrics (M2) results in fairer allocation than absolute fair mechanism $M 1$ and distance based mechanism M3 in both short term and long term fairness. The individual long term fairness is adopted to queue the request, therefore, it results in fairer individual allocation in CPE to CPE communication. However, the short term fairness is still much lower than the long term fairness, because the channels are very rare compared to amount of requests. For CPE to BS allocation, the amount of requests (on average more than 20 requests in a time slot) is much higher than the allocation rate (once in a time slot). Hence, both long term and short term CPE to BS fairness are very low, as shown in Fig. 2(d) and Fig. 2(c) 


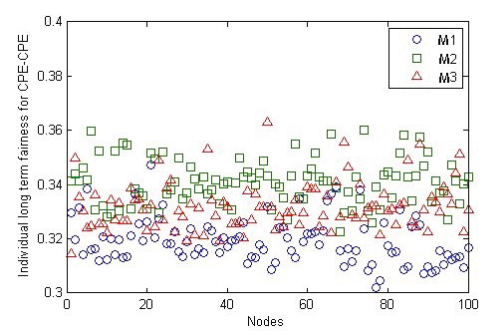

(a) Long term CPE to CPE fairness.

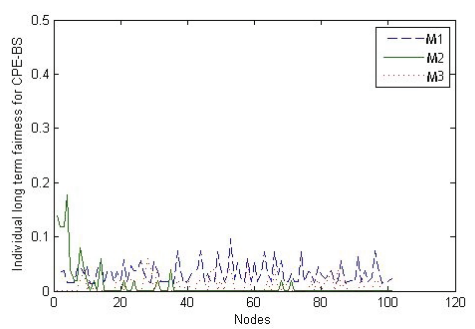

(c) Long term CPE to BS fairness.

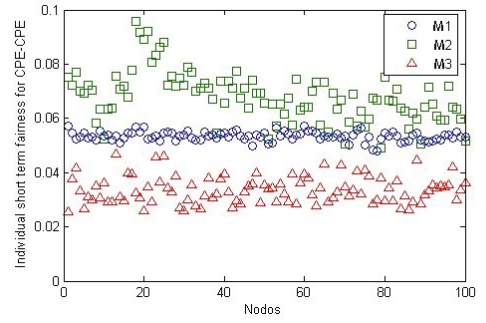

(b) Short term CPE to CPE fairness.

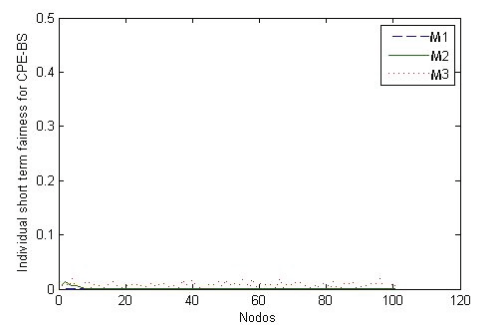

(d) Short term CPE to BS fairness.

Fig. 2. Individual fairness

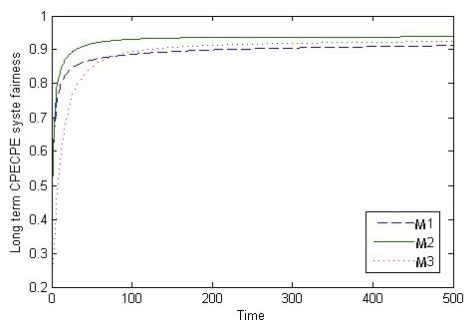

(a) Long term CPE to CPE fairness.

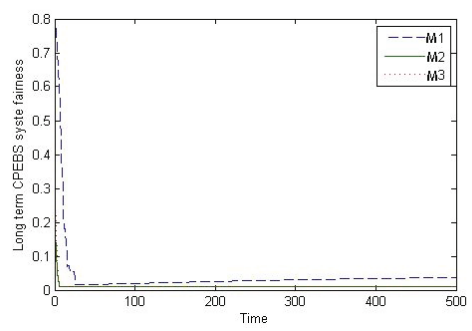

(c) Long term CPE to BS fairness.

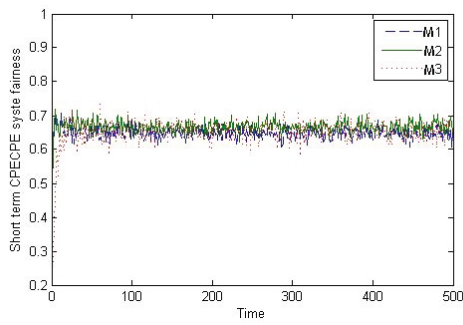

(b) Short term CPE to CPE fairness.

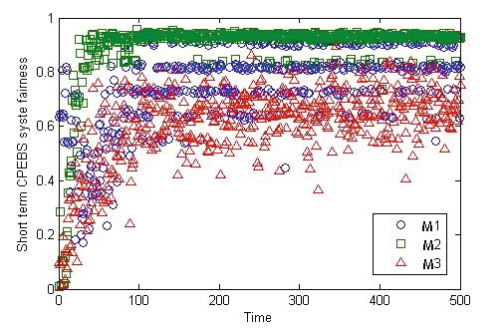

(d) Short term CPE to BS fairness.

Fig. 3. System fairness 
Results of system fairness are shown in Fig. 3 including both short term and long term system fairness. For CPE to CPE communication, long term fairness is adopted to queue the requests, therefore, mechanism (M2) leads to fairer allocation than $M 1$ and $M 3$ as shown in Fig. 3(a), The short term system fairness for CPE to CPE with M1, M2 and M3 are similar to each other as shown in Fig. 3(b), The long term system fairness for CPE to BS (Fig. 3(c) are very low because only one node can obtain a channel in one time slot, and the fairness for the rest is assigned to be 0 . The short term fairness for CPE to BS is increased by M2 (Fig. 3(d) because in many time slots there is no CPE to $\mathrm{BS}$ request, and the short term system fairness is assigned as 1 .

\section{Conclusions and Further Work}

Concerning all the resources and their allocation, fairness issue plays an important role in wireless networks. However, the metrics nowadays has many shortages. Therefore, a series of fairness metrics are proposed in this paper for resource allocation in wireless networks, which can measure the fairness of allocation from the point of view of individuals and system, further, also from the point view of short term and long term. We applied the developed fairness metric for IEEE 802.22 WRAN scenario. We also applied our metric to test the allocation fairness in a futuristic P2P channel usage in WRANs. The simulation results show that the metrics can be employed in wireless resource allocation mechanisms easily. When it is adopted in the channel allocation in P2PWRAN, higher fairness and performance can be achieved than the fairness and performance when absolute and distance based mechanisms are used. We are now planning to focus on more number of implementation scenarios (for example in rate allocation, flow and packet scheduling, power control and energy control) to test our metric, and testing its ability of measuring fairness.

\section{References}

1. Bertsekas, D.P., Gallager, R., Nemetz, T.: Data networks. Prentice-Hall, Englewood Cliffs (1987)

2. Jain, R., Chiu, D., Hawe, W.: A Quantitative Measure of Fairness and Discrimination for Resource Allocation in Shared Systems, Digital Equipment Corporation. Tech. rep., Technical Report DEC-TR-301 (1984)

3. Kelly, F., Maulloo, A., Tan, D.: Rate control for communication networks: shadow prices, proportional fairness and stability. The Journal of the Operational Research Society 49(3), 237-252 (1998)

4. Ko, G., Franklin, A., You, S., Pak, J., Song, M., Kim, C.: Channel management in IEEE 802.22 wran systems. IEEE Communications Magazine 48(9), 88-94 (2010)

5. Mo, J., Walrand, J.: Fair end-to-end window-based congestion control. IEEE/ACM Transactions on Networking (ToN) 8(5), 556-567 (2000)

6. Montuno, K., Zhacfi, Y.: Fairness of Resource Allocation in Cellular Networks: A Survey. In: Resource Allocation in Next Generation Wireless Networks, pp. 249 266 (2006) 
7. Radunovic, B., Le Boudec, J.Y.: A unified framework for max-min and min-max fairness with applications. IEEE/ACM Transactions on Networking 15(5), 10731083 (2007)

8. Shi, H., Prasad, R.R.V., Niemegeers, I.G.: An intra-cell peer to peer protocol in ieee 802.22 networks. In: GLOBALCOM (GC 2011) Workshop on Mobile Computing and Emerging Communication Networks (December 2011) (accepted)

9. Zheng, D., Zhang, J.: A two-phase utility maximization framework for wireless medium access control. IEEE Transactions on Wireless Communications 6(12), 4207-4299 (2007) 